\title{
Philosophiques
}

\section{La psychiatrie : une machine à faire croire}

\section{Roger Savoie}

Volume 4, numéro 2, octobre 1977

Philosophie et psychologie

URI : https://id.erudit.org/iderudit/203076ar

DOI : https://doi.org/10.7202/203076ar

Aller au sommaire du numéro

Éditeur(s)

Société de philosophie du Québec

ISSN

0316-2923 (imprimé)

1492-1391 (numérique)

Découvrir la revue

Citer cet article

Savoie, R. (1977). La psychiatrie : une machine à faire croire. Philosophiques, 4(2), 261-266. https://doi.org/10.7202/203076ar d'utilisation que vous pouvez consulter en ligne.

https://apropos.erudit.org/fr/usagers/politique-dutilisation/ 


\section{LA PSYCHIATRIE : UNE MACHINE À FAIRE CROIRE}

par Roger Savoie

Le sujet de mon intervention pourrait se résumer ainsi : le savoir psychiatrique joue le même rôle qu'une croyance religieuse et exerce sur les corps le même type de pouvoir. D'où l'opportunité de ne plus laisser la théorie guider la pratique et la possibilité d'expérimenter une forme de non-pensée et d'incroyance radicale.

J'éprouve un certain malaise à m'adresser à des gens qui pourraient se qualifier de professionnels de la maladie mentale. Mais comme il me semble évident aujourd'hui que le savoir psychiatrique est le fondement d'un pouvoir énorme qui déborde désormais les cadres de l'institution, je me sens ainsi habilité autant que n'importe qui, à titre d'observateur, d'acteur et de récepteur d'une pratique sociale bien ancrée, à en parler. Tout exercice de pouvoir m'apparât comme une réalité politique et idéologique. Politique veut dire ceci : un ensemble de forces conjuguées dans le but d'installer dans le corps et entre les corps une organisation cohérente et une discipline efficace. Idéologique veut dire que ce pouvoir est indissolublement uni à un savoir qui le fonde, mais aussi qu'il engendre et dont il est finalement le produit.

Je ne voudrais pas qu'on me prenne pourtant pour un défenseur de l'antipsychiatrie telle qu'elle s'est développée ces dernières années. Car l'antipsychiatrie, bien qu'elle se soit voulue une attaque fervente de la psychiatrie comme pouvoir politique, n'a pu s'exprimer qu'au sein de l'institution psychiatrique, en conservant le savoir essentiel de la science psychiatrique. Croyant innover, elle n'a été cependant capable d'exercer sa puissance destructrice qu'en s'accrochant par exemple à la doctrine phénoménologique, notamment à l'existentialisme sartrien pour qui toute conscience est projet d'être, dépassement de soi et se fonde sur l'expérience subjective du néant, c'est-à-dire sur le sentiment d'un manque fonda- 
mental. L'antipsychiatrie reste encore attachée à un savoir théorique, à uné pensée, et cet attachement prend dès lors tous les contours d'une croyance. Ce n'est là qu'un exemple.

Je ne vous dirai surtout pas qu'il appartient à la philosophie d'attaquer les fondements de la psychiatrie, car elle, autant et plus que tous les autres, serait la dernière à admettre que la pensée est croyante et à proposer une épreuve de non-pensée. Ce serait plutôt, si vous voulez me passer le mot, à une sorte d'exercice d'antiphilosophie que je pourrais me livrer devant vous.

En effet, c'est justement sur la citadelle de la pensée que pourrait porter dorénavant notre charge. Il semble paradoxal qu'il faille diriger notre attaque contre la pensée. La question que je pose est la suivante : se pourrait-il que nous ayons jusqu'ici, non pas mal pensé, car l'arsenal des concepts psychiatriques est réellement riche et impressionnant, mais que nous ayons trop pensé. Et si le savoir psychiatrique ne contribuait à la longue qu'à maintenir le malade dans une aire de savoir, dans l'idéologie de la prise de conscience, si nous nous en apercevions, nous pourrions nous mettre à faire autre chose, interrompre la pensée par exemple, ce qui pour moi serait l'équivalent d'interrompre la croyance en la pensée, nous pourrions peut-être essayer autre chose comme de recommencer à flairer, sentir, explorer sans la boussole de la théorie, douter de l'efficacité de notre savoir. Non pas tellement reprendre le doute cartésien à l'instar d'une pensée nouvelle qui s'inaugure et d'une conscience de soi qui s'éveille, mais douter que la pensée elle-même soit aussi forte qu'on le "pense " : son pouvoir est usurpé, elle ne semble pas pouvoir produire autre chose qu'un terrorisme, nous pourrions deviner dans ce savoir une reprise camouflée et plus insidieuse encore de l'immémoriale activité de la croyance.

Voilà pourquoi la psychiatrie m'apparaît comme une énorme machine à produire des énoncés de foi. Faire croi e voudrait dire ici : prétendre savoir quelque chose sur le corps de désir, créer l'illusion que nous pouvons décrypter le sens de ce qui s'y passe, réussir à opérer une sorte d'envahissement du corps par la pensée, faire croire que c'est elle qui nous délivrera, produire cette docilité, cette discipline, cet équilibre par lesquels chaque corps reproduirait de lui-même, par sa propre volonté (vouloir c'est pouvoir), par sa prise de conscience personnelle, un pouvoir interne, c'est-à-dire, un savoir qui le subjuguerait, le hanterait, le fascinerait, le rendrait 
actif et responsable et chargé de maturité. Faire croire à la longue que nous savons quelque chose sur le désir, que nous pouvons par conséquent le diriger. Faire croire par exemple que le corps s'éprouve fondamentalement comme manque, comme mécanique à besoins, comme lieu d'une insatisfaction essentielle, c'est-à-dire d'une maladie primitive, d'un drame ontologique ou historique. L'idéologie du manque deviendrait alors la pierre angulaire d'un pouvoir sur les corps, le pouvoir se définirait comme l'organisation systématique d'un régime de besoins, et sa satisfaction. Pour cela il suffit de créer l'illusion du vide intérieur, investir le corps par la pensée salutiste, au double sens du mot : prendre possession des corps et capitaliser sur eux. Si le corps est obsédé par son néant intérieur, s'il y croit, s'il adhère à ce savoir il serait alors tout disposé à se mettre au service d'une production sociale qui lui garantirait une plénitude venue de l'extérieur, il se soumettrait volontiers au pouvoir magique de celui qui veut sa guérison (et qui l'aura!), il deviendrait récupérable pour une plus belle participation au bien-être collectif.

Mais la théorie du manque n'est pas la seule qui pourrait être mise en doute. La psychiatrie a produit un nombre incalculable d'énoncés théoriques, base d'un nombre non moins admirable de pratiques thérapeutiques. Que dit-elle aux malades mentaux, à ces naufragés du sens? Elle dit, entre autres, qu'elle veut les aider à se retrouver, à rescaper leur identité, alors que c'est peut-être l'identité qu'il faut perdre, rejeter la théorie du moi, retrouver l'anonymat, cet anonymat que le pouvoir ne peut pas tolérer puisqu'il n'aurait plus de prise sur les corps individualisés, ce qui rendrait à chaque corps la possibilité de ne pas adhérer au projet social, de se singulariser en se multipliant, de changer d'identité au gré des événements, de n'avoir pas à être constamment étiqueté, quadrillé, lui permettrait de prononcer la formule de Lucifer, honnie et bannie parmi toutes : mon nom est légion, ou la formule léonéenne, mon nom est personne. Je ne suis personne parce que je suis trop de choses, mon rien ne débouche pas sur un vide intérieur mais sur un trop-plein. Incapable de devenir moi-même, je ne serais pas acculé à la névrose ou à la psychose. Devenir soi-même ressemble plus à un slogan publicitaire (d'ailleurs la publicité, avec son flair étonnant pour soumettre, $s$ 'en est emparé très vite), ressemble plus à une ruse du pouvoir qu'à une véritable prise en charge du soi. 
Que dit-elle encore la psychiatrie ? Elle prétend généralement que le malade doit retrouver un sens à la vie, une espérance pour l'avenir. Elle répète la croyance du patient, elle l'y enfonce. Elle ne lui dit pas : vous êtes peut-être malade de cette croyance, car la psychiatrie se veut respectueuse des croyances. Pourtant, il semble au moins probable que c'est de vouloir à tout prix prélever un sens à la vie que le malade a cru s'engouffrer dans l'insensé, que c'est d'avoir espéré qu'il est maintenant étouffé de désespoir. C'est que le thérapeute y croit, lui aussi, à ces vérités. Aux yeux d'un incroyant, le malade apparaît soudain comme un pion trié dans le tas, sélectionné au gré du hasard et des circonstances, selon des procédures illogiques mais nettement fonctionnelles, simple délégué de l'abstraction sociale, victime consentante et complice de la terreur théorique, cobaye du saint savoir, croyant parmi la foule des croyants, mais en quelque sorte plus croyant que les autres, choisi par l'institution sociale pour tracer les limites du désir. Des fous qui sont d'utilité publique et privée, producteurs de choses indispensables, producteurs de garde-fous, symboles vivants de ceux qui ont cru aux symboles et aux signes. Ils sont peut-être malades d'avoir trop cru au sens de la vie et trop espéré, malades d'avoir douté et de ne l'avoir pas supporté, malades du savoir, de ne pas savoir et de s'en croire diminués alors que les seuls modèles de conduite acceptables sont des modèles de la pensée correcte, de la pensée chercheuse, de celle qui cherche pour trouver. Avec eux, les fous, on est en sécurité, on sait que la pensée reste au pouvoir et que la non-pensée, le désir, le délire sont punis, séquestrés, démocratiquement, avec humanité, dans des conditions salubres. On sait jusqu'où ne pas aller, on est justifié de ses propres démissions, des étouffements secrets que notre propre conscience impose au désir. On n'ira pas boire à la coupe. Les malades sont devenus des modèles exemplaires. La sagesse croyante et savante définit dès lors les limites de sa propre folie et le thérapeute y trouve aussi son compte en y traçant les contours de sa propre sécurité. N'est-il pas vérifié quotidiennement que le thérapeute, même le moins directif, comme le sorcier antique, comme le prêtre d'il n'y a pas si longtemps, les attire infailliblement dans les régions lumineuses de la pensée?

Mais ne faudrait-il pas les juger moins sévèrement? Le dossier de l'affaire me semble en tout cas passablement accablant. La théorie et la théologie du manque, du moi et du sens ne sont pas 
les seules croyances auxquelles la psychiatrie accorde son adhésion de foi, mais aussi un nombre impressionnant d'idées, de buts, de valeurs, de principes qui tous se relient entre eux pour constituer le réseau fictif du besoin, rivant le croyant à son insatisfaction, à ses frustrations, formant ainsi un fardeau de vérités et $d$ 'habitudes mentales que le penseur porte héroïquement comme autant de prétextes à se courber, à jouir de sa bassesse. Il n'est pas possible ici de les énumérer toutes. Â titre d'exemples parmi tant d'autres, il croit encore, le penseur, au sujet libre et indépendant, à l'objet de la convoitise, à l'intentionnalité de la conscience, il croit à l'intériorité, et au subconscient, et au moi profond. Ou qu'il faut se donner un idéal dans la vie. Ou que toute chose doit avoir sa raison, que toute action doit avoir sa finalité, que tout phénomène est significatif et représentatif d'autre chose. Ou qu'il importe de se doter de valeurs épanouissantes, alors qu'on sait très bien entre nous que personne ne vit selon ses valeurs. Ou simplement qu'on doit profiter de la vie, jouir à tout prix, la mystique de la jouissance n'étant que le renversement de la mystique de l'ascèse. Ou qu'il faut devenir créateur, qu'on n'est pas sur terre pour rien. Ou que la subjectivité est enfouie dans l'objectivité de l'en-soi, dans la grande marmelade du monde. On dira encore : il suffit de satisfaire les besoins fondamentaux, besoins affectifs surtout, objets d'amour à investir, alors que l'expérience quotidienne et fragmentaire indique partout et à tous les échelons que chaque besoin fondamental est contredit par un besoin contraire non moins fondamental, par exemple que le besoin d'insécurité est aussi essentiel, normal, prioritaire, naturel, instinctif que le besoin de sécurité et de conservation, et que dès lors il n'y a pas de besoins fondamentaux, pas d'essence, pas de norme, pas de nature, pas de priorité, pas d'instinct, ou, si l'on veut, qu'il y en a trop, c'est indécidable, les codes sont brouillés, tout est chaotique, sans fondement, sans sujet et sans objet. On n'a finalement pas intérêt à trop prétendre en savoir làdessus. Ou encore on leur déclare; retournez en arrière, nous avons élaboré des méthodes pour que vous puissiez vous ressouvenir, revivre votre drame primitif, crier votre cri primal, trop longtemps étouffé, alors que sans doute il n'y a pas de drame primitif précis, pas de traumatisme particulier, pas de péché originel, c'est trop compliqué, il y a trop de sens et une forêt d'interprétations. La théorie du passé, comme toute théorie, peut devenir aussi totalitaire que les autres, il faudrait peut-être désapprendre cela, ne 
plus fragmenter le temps, apprendre à oublier le passé surtout, ou mieux encore dire: il n'y a pas de passé.

La psychiatrie comme science semble ainsi avoir poussé très loin l'aventure de la croyance alors qu'elle prétendait au contraire faire oeuvre de démythisation. Les psychiatres ont ainsi rendu leur propre incroyance incroyable, impossible, et, bien entendu, celle des malades. Elle a voulu se guider sur une vision du monde, de l'homme et de la société, elle s'est faite visionnaire, et prophétique, et cléricale. Elle a donné à l'acte théorique un crédit, un sérieux qui est le propre de tout homme au pouvoir avec ses qualités spécifiques: suractivité insomniaque, volonté de fer, organisation de buts et de moyens, déclaration de bonnes intentions, capacité de ritualisation. En soutenant ainsi le pouvoir, elle a rendu impossible une production anarchique de désirs et d'événements, d'expérimentations sans théorie, d'incroyance, de tâtonnements antiméthodiques. Elle a fait de son savoir une croyance. Voilà pourquoi un incroyant se mettrait à soupçonner que toute cette histoire de l'ordre du corps, de l'équilibre psychique, de la santé mentale n'est que la condition de l'ordre social. L'incroyant, parce qu'il éprouve autre chose, dirait qu'il n'y a pas de société, qu'on n'a donc pas à en être responsable, qu'on n'a pas à s'y adapter. S'adapter à la société c'est se soumettre encore une fois à une idée qu'on se fait d'elle. Le pouvoir sous toutes ses formes, politique, familial, pédagogique, psychiatrique, philosophique, n'est possible que s'il est installé solidement dans la pensée comme savoir. C'est au niveau des pouvoirs microscopiques que l'ensorcellement se fait, c'est donc ce pouvoir lui-même qu'un corps désirant veut détruire.

Nous ne pouvons plus maintenant faire semblant que le savoir est innocent et qu'il est pur. Personne ne peut plus nous faire croire à cela, à la neutralité et à l'objectivité du médecin. La volonté de vérité n'est jamais naïve et honnête. Il est à parier de plus en plus, au contraire, que le sublime idéal de connaître le vrai recouvre un projet, moins noble peut-être mais plus efficace, d'établir un pouvoir policier dans les corps et entre les corps.

Département de Philosophie, Cegep de St-Laurent. 